\title{
Rigid graph-based three-dimension localization algorithm for wireless sensor networks
}

\author{
LUO Xiaoyuan ${ }^{1, *}$, ZHONG Wenjing $^{1}$, LI Xiaolei ${ }^{1}$, and GUAN Xinping ${ }^{2}$
}

1. School of Electrical Engineering, Yanshan University, Qinhuangdao 066004, China;

2. Department of Automation, Shanghai Jiao Tong University, Shanghai 200240, China

\begin{abstract}
This paper investigates the node localization problem for wireless sensor networks in three-dimension space. A distributed localization algorithm is presented based on the rigid graph. Before location, the communication radius is adaptively increasing to add the localizability. The localization process includes three steps: firstly, divide the whole globally rigid graph into several small rigid blocks; secondly, set up the local coordinate systems and transform them to global coordinate system; finally, use the quadrilateration iteration technology to locate the nodes in the wireless sensor network. This algorithm has the advantages of low energy consumption, low computational complexity as well as high expandability and high localizability. Moreover, it can achieve the unique and accurate localization. Finally, some simulations are provided to demonstrate the effectiveness of the proposed algorithm.
\end{abstract}

Keywords: wireless sensor network, localization, rigid graph, quadrilateration.

DOI: $10.21629 / J S E E .2018 .05 .05$

\section{Introduction}

Recently, wireless sensor networks (WSNs) have a wide spectrum of applications, such as environment monitoring, disaster prevention and smart grid information surveillance [1-5], because of the advantages of low-cost, low-power and tiny in size. At the same time, the localization problem for WSNs becomes a significant issue attracting considerable research interests [6-8].

Based on the available measurement data type, the localization algorithms are typically divided into three kinds: the angle-based, the distance-based and the received signal strength (RSS)-based [9-13]. Angle-based localization measures the direct line of sight path from the transmitter to the receiver. The accuracy of the measurements is limited by the directivity of the antenna, by shadowing and

\footnotetext{
Manuscript received September 04, 2017.

*Corresponding author.

This work was supported by the National Natural Science Foundation of China (61375105; 61403334).
}

by multipath reflections [14]. Distance-based localization measures the difference between a signal's sending time at the transmitter and this signal's receiving time at the receiver, then obtains the distance on the basis of the time difference and signal propagation velocity. It requires the synchronization between transmitter and receiver, which makes it hard to deploy $[9,15]$, but the accuracy of this measurement is higher than others. RSS-based localization measures the attenuation degree of the signal strength, then constructs a form of map of the signal strength in the deployment area. This approach does not need clock synchronization but the RSSI values are generally corrupted by environment noise [14,16], especially in three-dimensional situation. In this paper, we use the distance-based localization algorithm for WSNs.

In [17], David Moore et al. proposed an algorithm that can localize nodes in a sensor network using the distance information estimated by each of its neighbors. The algorithm consists of three phases which are cluster localization, cluster optimization and cluster transformation. In [18], a WSNs localization algorithm was presented by using the fixed and mobile guide nodes. In this paper, nearly $20 \%$ of nodes are fixed guide nodes and three nodes are intended as mobile guide nodes. In [19], a new graph embedding method based on polynomial mapping was proposed. In [20], a geometric triangulation scheme which is an alternative way of calculating the estimated position of the object was proposed based on the angle of arrival between an object node and its references. These algorithms are all applied in 2-dimensional (2D) space. In fact, in practical applications, such as underwater environment, the deployment of sensor network is in 3-dimensional (3D) space rather than in 2D space. What's more, located nodes in 3D space does not simply increase an extra dimension to the localization problem, because compared with 2D space, the environment of the practical applications and node position calculation are more complex in the 3D space, so 
that it is necessary to develop 3D localization technology for WSNs broadly.

In [21], a 3D target localization algorithm in noncooperative WSNs was presented by constructing a novel objective function for the hybrid localization problem based on the least squares criterion. In [22], a 3D localization algorithm for WSNs based on particle swarm optimization was presented. In [23], a 3D localization technique based on the hop-count data was proposed to estimate the location of the activated sensor in $3 \mathrm{D}$ coordination. This localization technique can guarantee both improved accuracy and power efficiency. However, they did not consider the influence of nodes' communication radius to localization, and did not choose the appropriate communication radius.

In this paper, we present a distributed and distancebased localization algorithm by utilizing rigid graph for large scale 3D WSNs. Before location, the communication radius is adaptively increasing to add the localizability. This localization algorithm uses rigid blocks division and iterative quadrilateration mainly to realize the localization for the entire network nodes. Compared with some centralized algorithm [24-27], we find that this algorithm has less energy consumption, low computational complexity and high expandability. Moreover, it can achieve the unique and accurate localization.

The reminder of this paper is organized as follows: in Section 2, some necessary preliminaries about rigid graph theory and quadrilateration are introduced. In Section 3, a localization algorithm for WSNs based on rigid graph is presented. The simulation results are provided in Section 4. Section 5 concludes this paper.

\section{Preliminary}

In this section, we will introduce some basic concepts of algebraic graph theory and quadrilateration theory.

\subsection{Graph theory and rigid graph-based localization}

A $d$-dimensional $(d \in 2,3)$ formation at $\boldsymbol{p}=$ $\left(\boldsymbol{p}_{1}, \boldsymbol{p}_{2}, \ldots, \boldsymbol{p}_{N}\right)$ is usually denoted as $\boldsymbol{F}(\boldsymbol{p}), N$ is the number of nodes and $\boldsymbol{p}_{i}(i=1,2, \ldots, N) \in \mathbf{R}^{d}$ is the location of node $i$. There is a communication link between $i$ and $j$ if there is an interaction between them, the distance of this communication link is called Euclidean distance and expressed as $d_{i j}$. Some basic definitions and lemmas are given below.

Definition 1 (Sensor graph [28]) A WSN $N(S, D)$, where $S$ and $D$ represent the WSN node set and the distance set respectively, can be described by a graph $G=$ $(V, E)$ with the vertex set $V=\{1,2, \ldots, N\}$ and an edge set $E=\left\{(i, j) \mid d_{i j}>0, i, j \in V\right\}$. Each vertex $i \in V$ is related to a sensor $s_{i} \in S$ in the WSN and each edge $(i, j) \in E$ is related to a pair of sensors $\left(s_{i}, s_{j}\right)$. We call $G=(V, E)$ the underlying graph of the sensor network $N(S, D)$.

Definition 2 (Framework and realization [29,30]) A $d$-dimensional $(d \in 2,3)$ expression of graph is mapping this graph $G=(V, E)$ to a formation $\boldsymbol{F}(\boldsymbol{p}): V \rightarrow \mathbf{R}^{d}$. Given graph $G=(V, E)$ and its $d$-dimensional expression, the pair $(G, \boldsymbol{p})$ is termed as a $d$-dimensional framework. The distance set $D$ for $G$ is the set of distances for all edges $(i, j) \in E$. Given the distance set $D$ for the graph $G$, a $d$-dimensional $(d \in 2,3)$ expression $\boldsymbol{p}$ of $G$ is a realization if $\left\|\boldsymbol{p}_{i}-\boldsymbol{p}_{j}\right\|=d_{i j}$ for all pairs of $(i, j) \in E$.

Definition 3 (Equivalent and congruent frameworks $[29,30])$ Two frameworks $(G, \boldsymbol{p})$ and $(G, \boldsymbol{q})$ are equivalent if $\left\|\boldsymbol{p}_{i}-\boldsymbol{p}_{j}\right\|=\left\|\boldsymbol{q}_{i}-\boldsymbol{q}_{j}\right\|$ holds for every pair of $(i, j) \in E$. Two frameworks $(G, \boldsymbol{p})$ and $(G, \boldsymbol{q})$ are congruent if $\left\|\boldsymbol{p}_{i}-\boldsymbol{p}_{j}\right\|=\left\|\boldsymbol{q}_{i}-\boldsymbol{q}_{j}\right\|$ holds for every pair of $(i, j)$, no matter whether there is a connection between them.

Definition 4 (Flexible graph and rigid graph [30,31]) A framework $(G, \boldsymbol{p})$ is called generic if the coordinates of all nodes are algebraically independent. A framework $(G, \boldsymbol{p})$ is called rigid if there exists a sufficiently small positive constant $\varepsilon$ such that if $(G, \boldsymbol{p})$ is equivalent to $(G, \boldsymbol{q})$ and $\left\|\boldsymbol{p}_{i}-\boldsymbol{q}_{i}\right\|<\varepsilon$ for all $i \in V$, then $(G, \boldsymbol{p})$ is congruent with $(G, \boldsymbol{q})$. A graph $G=(V, E)$ is called rigid if there is an associated framework $(G, \boldsymbol{p})$ that is generic and rigid. A framework is called flexible if one has a continuous deformation starting from the known configuration to another, and the edge lengths are remained, an example is shown in Fig. 1(a) and Fig. 1(b). If such deformation does not exist, it is called rigid. A framework $(G, \boldsymbol{p})$ is called globally rigid if every framework equivalent to $(G, \boldsymbol{p})$ is also congruent with $(G, \boldsymbol{p})$. A graph $G=(V, E)$ is called generically globally rigid (GGR) if there is an associated framework $(G, \boldsymbol{p})$ that is generic and globally rigid.

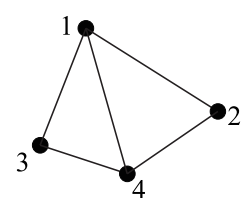

(a) A flexible framework

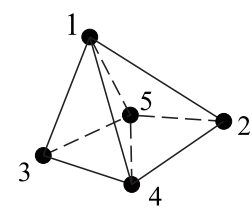

(c) A minimally rigid framework

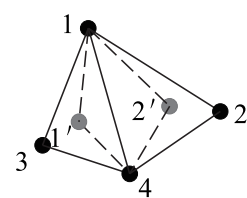

(b) A deformation from (a)

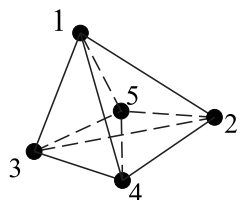

(d) A redundantly rigid framework
Fig. 1 Several frameworks

Definition 5 (Minimal rigid framework [31]) A rigid 
framework is minimally rigid if it becomes flexible after removing an arbitrary edge, an example is shown in Fig. 1(c).

Definition 6 (Redundantly rigid framework [30]) A rigid framework is redundantly rigid if removing any edge of $G$ results in a rigid graph, an example is shown in Fig. 1(d).

Definition 7 (Localizability [32]) A graph $G=(V, E)$ with the conditions of several nodes locations being known is called localizable if there is a unique location for each node in WSNs such that the $d_{i j}=\left\|\boldsymbol{p}_{i}-\boldsymbol{p}_{j}\right\|$ for all edges $(i, j) \in E$ and the conditions are remained. Different from localization that calculates the locations of nodes in WSNs, localizability focuses on the location-uniqueness of nodes in WSNs.

Lemma 1 [28] Let $N(S, D)$ be a sensor network graph in $d$-dimensional. Let $(G, \boldsymbol{p})$ be the framework for the underlying graph $G(V, E)$. Then the sensor network localization problem is solvable only if $(G, \boldsymbol{p})$ is generically globally rigid.

Lemma 2 [30] Let $G$ be a globally rigid graph with at least $(d+1)$ vertices in $\mathbf{R}^{d}(d \in 2,3)$. Then the graph $G$ is $(d+1)$-connected, and redundantly rigid in $\mathbf{R}^{d}$.

\subsection{Quadrilateration}

We can obtain the locations of all nodes within the generically globally rigid graphs using the notion of quadrilateration in $\mathbf{R}^{3}$. In other words, consider a graph $G=(V, E)$ in $\mathbf{R}^{3}$, using quadrilateration on the GGR graph $G$, one can add the number of vertices with known locations. In the initial state, there must be at least four nodes which are regarded as anchor nodes.

Definition 8 (Quadrilateration graphs [33]) A quadrilateration graph $G=(V, E)$ in $\mathbf{R}^{3}$ is the set of ordered vertices $\left(v_{1}, v_{2}, \ldots, v_{N}\right)$, in which the first four vertices are regarded as anchor nodes and the edges $\left(v_{1}, v_{2}\right),\left(v_{1}, v_{3}\right)$, $\left(v_{1}, v_{4}\right),\left(v_{2}, v_{3}\right),\left(v_{2}, v_{4}\right),\left(v_{3}, v_{4}\right)$ are all presented in edge set $E$ initially. In addition, each vertex $v_{i}$ for $i$ varying from $i=5,6, \ldots, N$ remains connected to four of the vertices $v_{1}, v_{2}, \ldots, v_{i-1}$. The quadrilateration graph can be obtained by using consecutive quadrilateration procedure for the primal vertices $v_{1}, v_{2}, v_{3}, v_{4}$ and the edges between them.

\section{Main result}

The generic process of WSNs' nodes localization using rigid graph concepts is depicted in Fig. 2, which contains the following phases:

(i) Nodes deployment. In this phase, the nodes of the WSNs are deployed randomly in the deployment area.

(ii) Rigid judgement. In this phase, rigidity of the above graph is judged. It is necessary to increase the communication radius adaptively to make the graph become globally rigid. As a result, the initial deployment of the WSN nodes will turn to a new WSN deployment.

(iii) Rigid blocks division. In this phase, the minimum rigid blocks are obtained. The detailed approach is shown in the subsection of rigid block formation.

(iv) Local coordinate system establishment. In this phase, the local coordinate systems of the obtained blocks are introduced in the subsection of local coordinate system.

(v) Global coordinate system transformation. In this phase, the blocks convert the local coordinate system to global coordinate system by using rotation, translation and mirror if necessary. The details are explained in the subsection of global transformations.

(vi) Iterative quadrilateration. Quadrilateration of the rigid blocks will generate the location estimation of the unknown nodes. The details are introduced in the subsection of the localization procedure.

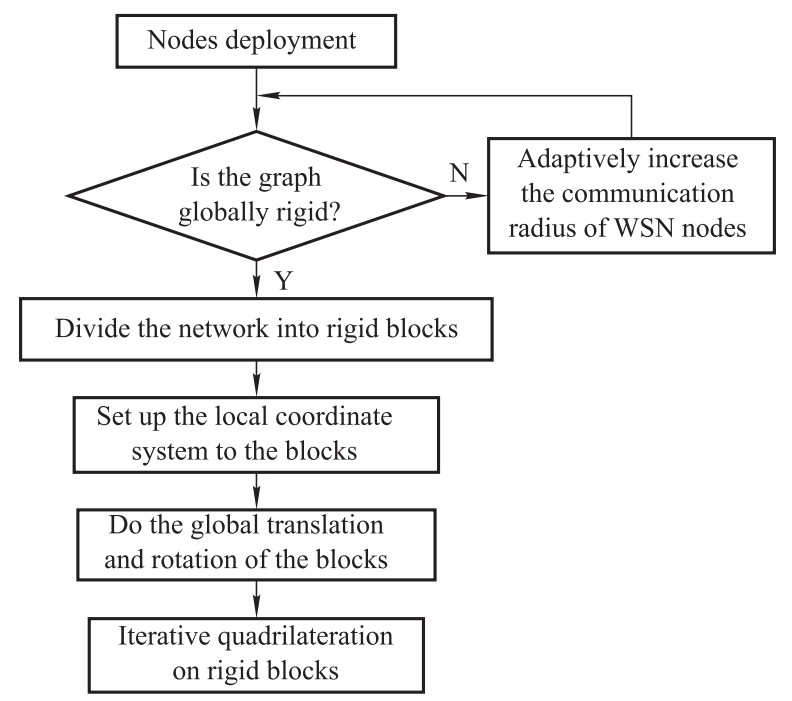

Fig. 2 Process of localization

\subsection{Rigid block formation}

In the practical applications, since the original WSN graph is always complicated, it is necessary to decompose the given WSN graph to minimally rigid graph which is easy to express by simple formulas. In $\mathbf{R}^{3}$, the minimally rigid block is tetrahedron. In this paper, the whole WSN graph would be decomposed to tetrahedrons. In this way, the complicated graph can be simplified efficiently. In the WSN graph, the nodes have general characteristics as follows: each WSN node has its own ID and knows the distances to its neighbors. The two nodes at the terminal of the communication link are interactional to send or receive message from each other. 
The adjacency matrix is a significant tool to represent the connection relation between nodes. We can judge whether there exists an edge between two nodes or not by using the adjacency matrix. Therefore, it is supposed to construct the adjacency matrix in terms of the given WSN graph in original. The process to find minimally rigid graph is described briefly as follows.

In $\mathbf{R}^{3}$, there are two steps to search tetrahedron. At first, we should find a triangle, then check a node in the WSN which is connected with each vertex of the previous triangle. In the first step, we put the ID of the first node into a matrix named $C \boldsymbol{Y C L E}$ initially. Then find its 1-hop neighbor and put the neighbor's ID into $C Y C L E$, we call it a pace, denoted by pace $=1$. Repeat the operation until pace $=3$ and the ID that we put into $C \boldsymbol{Y C L E}$ conclusively is the same as the initial ID, then a triangle is found. In the next step, we go on to find a node $j$ which is the 1-hop neighbor of all the vertices of the previous triangle and it must not be coplanar with the triangle. In this way, a smallest rigid component, the tetrahedron which is composed of the previous triangle and node $j$, is obtained. Finally, we put this tetrahedron with its NODE-ID in a matrix called RIGID. Continue doing these two steps repeatedly until all the tetrahedrons, which are the smallest rigid components of the WSN graph, are found out.

\subsection{Local coordinate system}

Each block decomposed in the above subsection has its own local coordinate system. In Fig. 3(a), two different coordinate systems, by putting $\boldsymbol{P}_{1}$ and $\boldsymbol{P}_{2}$ as the origins, respectively, are shown. It is clear that the coordinate system $\left(x_{1}, y_{1}, z_{1}\right)$ is located at $\boldsymbol{P}_{1}$, while the coordinate system $\left(x_{2}, y_{2}, z_{2}\right)$ is located at $\boldsymbol{P}_{2}$. In $\mathbf{R}^{3}$, there are two angles $\theta_{i j}$ and $\varphi_{i j}(i, j=1,2)$, which is different from $\mathbf{R}^{2}$ with only one angle. $\theta_{i j}$ is the angle between the $z_{i}$-axis and the connected link as well as $\varphi_{i j}$ is the angle between the $x_{i}$ axis and the projection of connected link in the $x_{i} o y_{i}$-plane in the local coordinate system. Also, these two angles are measured with an anticlockwise direction from the $z_{i}$-axis or $x_{i}$-axis of the WSN nodes local coordinate system similarly, but $\theta_{i j}$ is ended to communication link while $\varphi_{i j}$ is ended to the projection of connected link in the $x_{i} o y_{i}$ plane. In the local coordinate system of $\boldsymbol{P}_{1}$, the coordinate of $\boldsymbol{P}_{2}$ can be expressed by using $\theta_{12}$ and $\varphi_{12}$. In the same way, the coordinate of $\boldsymbol{P}_{1}$ can be expressed by using $\theta_{21}$ and $\varphi_{21}$ in the local coordinate system of $\boldsymbol{P}_{2}$.

A block decomposed in the previous subsection is considered, and it has four nodes $\boldsymbol{P}_{1}, \boldsymbol{P}_{2}, \boldsymbol{P}_{3}$, and $\boldsymbol{P}_{4}$ whose coordinates are $\left(x_{1}, y_{1}, z_{1}\right),\left(x_{2}, y_{2}, z_{2}\right),\left(x_{3}, y_{3}, z_{3}\right)$, and $\left(x_{4}, y_{4}, z_{4}\right)$, respectively. As shown in Fig. 3(b), $\boldsymbol{P}_{1}$ is the coordinate origin, $\boldsymbol{P}_{2}, \boldsymbol{P}_{3}$, and $\boldsymbol{P}_{4}$ are all called the 1-hop neighbor of $\boldsymbol{P}_{1}$ because there is a direct communication link between $\boldsymbol{P}_{1}$ and $\boldsymbol{P}_{i}(i=2,3,4)$. To construct the local coordinate system of each block, it is necessary to know the distance $d_{i j}$ between any two nodes and the corresponding angles $\theta_{i j}$ and $\varphi_{i j}$.

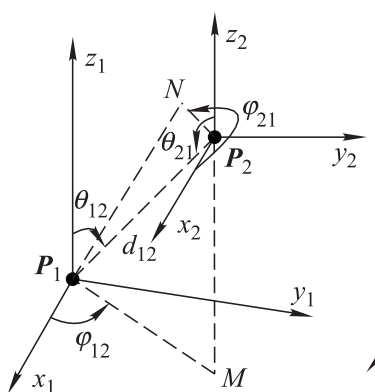

(a) Two different coordinate systems

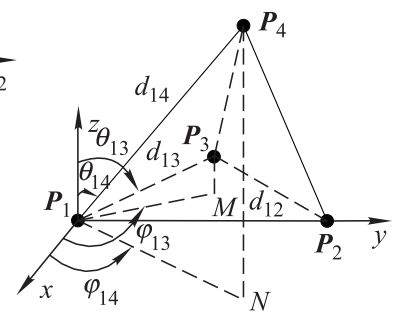

(b) A local coordinate system of a block
Fig. 3 Local coordinate systems of WSN nodes

In Fig. 3(b), the coordinates of $\boldsymbol{P}_{1}, \boldsymbol{P}_{2}, \boldsymbol{P}_{3}$, and $\boldsymbol{P}_{4}$ are shown as follows:

$$
\begin{gathered}
\left\{\begin{array}{l}
P_{1_{X}}=0 \\
P_{1_{Y}}=0, \quad\left\{\begin{array}{l}
P_{2_{X}}=0 \\
P_{2_{Y}}=d_{12} \\
P_{1_{Z}}=0
\end{array},\right.
\end{array},\right. \\
\left\{\begin{array}{l}
P_{3_{X}}=d_{13} \sin \theta_{13} \cos \varphi_{13} \\
P_{3_{Y}}=d_{13} \sin \theta_{13} \sin \varphi_{13} \\
P_{3_{Z}}=d_{13} \cos \theta_{13}
\end{array}\right. \\
\left\{\begin{array}{l}
P_{4_{X}}=d_{14} \sin \theta_{14} \cos \varphi_{14} \\
P_{4_{Y}}=d_{14} \sin \theta_{14} \sin \varphi_{14} \\
P_{4_{Z}}=d_{14} \cos \theta_{14}
\end{array}\right.
\end{gathered}
$$

with

$$
\begin{aligned}
& \left\{\begin{array}{l}
\theta_{13}=\arccos \frac{P_{3_{Z}}^{2}+d_{13}^{2}-P_{3_{X}}^{2}-P_{3_{Y}}^{2}}{2 d_{13} P_{3_{Z}}} \\
\varphi_{13}=\frac{\pi}{2}+\arccos \frac{P_{3_{Y}}}{\sqrt{P_{3_{X}}^{2}+P_{3_{Y}}^{2}}}
\end{array}\right. \\
& \left\{\begin{array}{l}
\theta_{14}=\arccos \frac{P_{4_{Z}}^{2}+d_{14}^{2}-P_{4_{X}}^{2}-P_{4_{Y}}^{2}}{2 d_{14} P_{3_{Z}}} \\
\varphi_{14}=\arccos \frac{P_{4_{Y}}}{\sqrt{P_{4_{X}}^{2}+P_{4_{Y}}^{2}}}
\end{array} .\right.
\end{aligned}
$$

By solving (1), the angles $\theta_{13}, \varphi_{13}$ and coordinates $P_{3_{X}}, P_{3_{Y}}, P_{3_{Z}}$ can be obtained. Similarly, the angles $\theta_{14}$, $\varphi_{14}$ and coordinates $P_{4_{X}}, P_{4_{Y}}, P_{4_{Z}}$ can be obtained.

\subsection{Global transformation}

As the directions of two local coordinate systems may be inconsistent, it is necessary to do some directional analysis and transformation for different local coordinate systems in order to obtain the global coordinate system, which can 
estimate the actual physical location for each WSN node by using four nodes in this network.

There are two possible situations in the process of global coordinate systems transformation. In the first situation, the coordinate system directions of $\boldsymbol{P}_{1}$ and $\boldsymbol{P}_{2}$ are consistent, that is, the coordinate system of $\boldsymbol{P}_{2}$ can be converted to the coordinate system of $\boldsymbol{P}_{1}$ by using coordinate translation and rotation. In the second situation, coordinate translation and rotation cannot make the coordinate systems $\boldsymbol{P}_{2}$ and $\boldsymbol{P}_{1}$ have the same direction, thus, the coordinate system of $\boldsymbol{P}_{2}$ needs to be mirrored around one of its coordinate plane after translation and rotation.

In the first situation, to localize the ordinary node, the global transformation matrix $\boldsymbol{M}_{G}$ translates local coordinate to global coordinate system. The global transformation matrix $\boldsymbol{M}_{G}$ includes translation matrix $\boldsymbol{T}_{G}$ and rotation matrix $\boldsymbol{R}_{G}$. As shown in Fig. 4, the local coordinate system of $\boldsymbol{P}_{1}$ is regarded as the global coordinate system.

Thus, the translation matrix $\boldsymbol{T}_{G}$ is derived from the translation of the local coordinate system of $\boldsymbol{P}_{2}$ to the global coordinate system, as shown in Fig. 4(a). In Fig. 4(a), $\Delta x, \Delta y, \Delta z$ are the components of the translation distance in $x$-axis, $y$-axis, $z$-axis, respectively. Through the translation, the two origin points intersect at point $O$. Rotation matrix $\boldsymbol{R}_{G}$ is derived from the rotation of the local coordinate system to the global coordinate system, and there are three rotations which are rotated around $x$-axis, around $y$-axis, around $z$-axis, respectively. An example of the rotation process is shown in Fig. 4(b), Fig. 4(c) and Fig. 4(d). Because the local coordinate system of $\boldsymbol{P}_{1}$ is regarded as the global coordinate system, its three axes are called $x$-axis, $y$-axis and $z$-axis, respectively. It is supposed to convert the coordinate system of $\boldsymbol{P}_{2}$ to the coordinate system of $\boldsymbol{P}_{1}$. In Fig. 4(b), $\alpha$ is the angle between $z$-axis and the projection of $z_{2}$-axis to yoz-plane, $z_{2}$-axis rotates according to the direction as shown in Fig. 4(b). The result is shown in Fig. 4(c). Now $z_{2}$-axis is in the xoz-plane. $\beta$ is the angle between $z$-axis and $z_{2}$-axis, through the rotation, the result is shown in Fig. 4(d). Rotate again in terms of $\gamma$, these two coordinate systems become consistent. In the same way, through the rotation, the local coordinate systems of all rigid blocks convert to the global coordinate system. The global transformation matrix $\boldsymbol{M}_{G}$ is

$$
\left[\boldsymbol{M}_{G}\right]=\left[\boldsymbol{T}_{G}\right] \cdot\left[\boldsymbol{R}_{G}\right]
$$

where

$$
\boldsymbol{T}_{G}=\left[\begin{array}{cccc}
1 & 0 & 0 & 0 \\
0 & 1 & 0 & 0 \\
0 & 0 & 1 & 0 \\
\Delta x & \Delta y & \Delta z & 1
\end{array}\right],
$$

$$
\begin{gathered}
\boldsymbol{R}_{G}=\left[\begin{array}{cccc}
1 & 0 & 0 & 0 \\
0 & \cos \alpha & \sin \alpha & 0 \\
0 & -\sin \alpha & \cos \alpha & 0 \\
0 & 0 & 0 & 1
\end{array}\right] . \\
{\left[\begin{array}{cccc}
\cos \beta & 0 & -\sin \beta & 0 \\
0 & 1 & 0 & 0 \\
\sin \beta & 0 & \cos \beta & 0 \\
0 & 0 & 0 & 1
\end{array}\right] .} \\
{\left[\begin{array}{cccc}
\cos \gamma & \sin \gamma & 0 & 0 \\
-\sin \gamma & \cos \gamma & 0 & 0 \\
0 & 0 & 1 & 0 \\
0 & 0 & 0 & 1
\end{array}\right] .}
\end{gathered}
$$

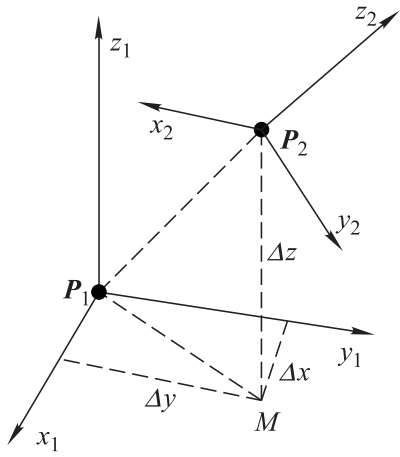

(a) Translation $\left(\boldsymbol{P}_{2} \rightarrow \boldsymbol{P}_{1}\right)$

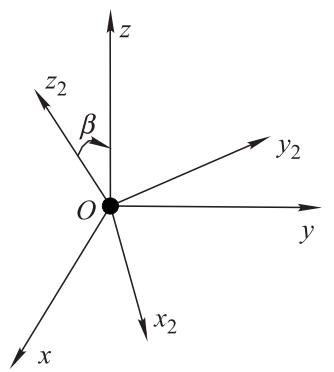

(c) Rotating around $y$-axis

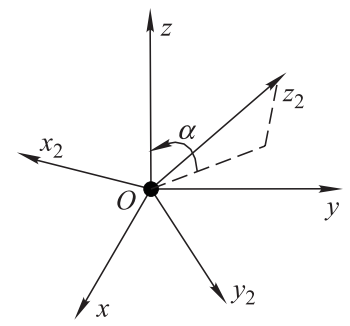

(b) Rotating around $x$-axis
Fig. 4 An example of coordinate system transformation

In the second situation, through coordinate translation and rotation, a mirrored coordinate system appears. An example is shown in Fig. 5. The two coordinate systems are symmetrical with respect to xoz-plane, then the global coordinate system is obtained by multiplying mirror symmetric matrix $\boldsymbol{M \boldsymbol { I } _ { G }}$. In other words, the global transformation matrix $\boldsymbol{M}_{G}$ includes translation matrix $\boldsymbol{T}_{G}$, rotation matrix $\boldsymbol{R}_{G}$ and mirror symmetric matrix $\boldsymbol{M} \boldsymbol{I}_{G}$. The global transformation matrix $\boldsymbol{M}_{G}$ is

$$
\left[\boldsymbol{M}_{G}\right]=\left[\boldsymbol{T}_{G}\right] \cdot\left[\boldsymbol{R}_{G}\right] \cdot\left[\boldsymbol{M} \boldsymbol{I}_{G}\right]
$$

where $\boldsymbol{T}_{G}$ and $\boldsymbol{R}_{G}$ are the same as the first situation, and $\boldsymbol{M} \boldsymbol{I}_{G}$ in this example is shown as follows: 


$$
\left[\boldsymbol{M I}_{G}\right]=\left[\begin{array}{cccc}
1 & 0 & 0 & 0 \\
0 & -1 & 0 & 0 \\
0 & 0 & 1 & 0 \\
0 & 0 & 0 & 1
\end{array}\right] .
$$

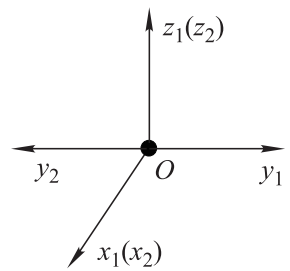

Fig. 5 An example of mirrored coordinate system as mentioned in the second situation

In any situation mentioned above, local coordinate system can convert to global coordinate system eventually. Once all local coordinate systems in WSN turn to global coordinate system via translation, rotation and mirror symmetric if necessary, it is the time to locate the nodes in this WSN.

\subsection{Localization procedure}

As is known for us, if three nodes are not collinear and the lengths of three edges which connect these three nodes with object nodes are known, we can locate a unique object node in 2D. Similarly, if four nodes are not coplanar and the lengths of four edges which connect these four nodes with object nodes are known, we can locate a unique object node in 3D, it is called quadrilateration. By using iterative quadrilateration on the basis of rigid blocks with global coordinate obtained in the subsection of local coordinate system and the subsection of global transformation, the localization is done.

We use an example shown as Fig. 6 to depict the operations. In order to simplify calculation, without loss of generality, one of the referenced nodes is translated to origin, denote

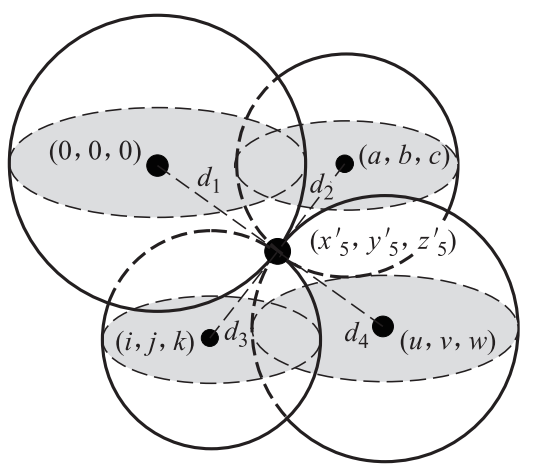

Fig. 6 Three-sphere intersection based quadrilateration

$$
\begin{gathered}
\left\{\begin{array}{l}
a=x_{2}-x_{1} \\
b=y_{2}-y_{1} \\
c=z_{2}-z_{1}
\end{array}, \quad\left\{\begin{array}{l}
i=x_{3}-x_{1} \\
j=y_{3}-y_{1}, \\
k=z_{3}-z_{1}
\end{array}\right.\right. \\
\left\{\begin{array}{l}
u=x_{4}-x_{1} \\
v=y_{4}-y_{1} \\
w=z_{4}-z_{1}
\end{array}, \quad\left\{\begin{array}{l}
x_{5}^{\prime}=x_{5}-x_{1} \\
y_{5}^{\prime}=y_{5}-y_{1} \\
z_{5}^{\prime}=z_{5}-z_{1}
\end{array} .\right.\right.
\end{gathered}
$$

In Fig. 6, the big dark circled point is the unknown coordinate $\left(x_{5}^{\prime}, y_{5}^{\prime}, z_{5}^{\prime}\right)$ whose position has to be estimated based on the known coordinates (small dark circled points). $d_{1}, d_{2}, d_{3}$ and $d_{4}$ are the known communication radius between the referenced nodes and the object node.

The coordinates of four referenced nodes can be expressed as $(0,0,0),(a, b, c),(i, j, k)$ and $(u, v, w)$. According to the geometrical relationship, the distances are given by

$$
\left\{\begin{array}{l}
d_{1}^{2}=x_{5}^{\prime 2}+y_{5}^{\prime 2}+z_{5}^{\prime 2} \\
d_{2}^{2}=\left(x_{5}^{\prime}-a\right)^{2}+\left(y_{5}^{\prime}-b\right)^{2}+\left(z_{5}^{\prime}-c\right)^{2} \\
d_{3}^{2}=\left(x_{5}^{\prime}-i\right)^{2}+\left(y_{5}^{\prime}-j\right)^{2}+\left(z_{5}^{\prime}-k\right)^{2} \\
d_{4}^{2}=\left(x_{5}^{\prime}-u\right)^{2}+\left(y_{5}^{\prime}-v\right)^{2}+\left(z_{5}^{\prime}-w\right)^{2}
\end{array} .\right.
$$

By solving (5), the relational expression is obtained as follows:

$$
\begin{gathered}
\left\{\begin{array}{l}
x_{5}^{\prime}=C_{1}-\frac{b}{a} y_{5}^{\prime}-\frac{c}{a} z_{5}^{\prime} \\
y_{5}^{\prime}=C_{2}-\frac{i}{j} x_{5}^{\prime}-\frac{k}{j} z_{5}^{\prime} \\
z_{5}^{\prime}=C_{3}-\frac{u}{w} x_{5}^{\prime}-\frac{v}{w} y_{5}^{\prime}
\end{array}\right. \\
\left\{\begin{array}{l}
C_{1}=\frac{d_{1}^{2}-d_{2}^{2}+a^{2}+b^{2}+c^{2}}{2 a} \\
C_{2}=\frac{d_{1}^{2}-d_{3}^{2}+i^{2}+j^{2}+k^{2}}{2 j} \\
C_{3}=\frac{d_{1}^{2}-d_{4}^{2}+u^{2}+v^{2}+w^{2}}{2 w}
\end{array}\right.
\end{gathered}
$$

Solving (6), the coordinate information of the unique point $\left(x_{5}, y_{5}, z_{5}\right)$ could be obtained. In this way, the number of already located nodes is increasing. Next, choose any four nodes whose coordinates have been known and repeat the above quadrilateration operation, all node coordinates in WSN can be obtained.

\section{Simulations}

In this section, several simulations are made to quantitatively analyze and validate the effectiveness of the proposed algorithm.

The simulations reveal the variety of node density, average node degree and the number of localization nodes as well as the relationship between them. There are several simulation parameters as shown in Table 1 , and $\mathrm{n}$ denotes 
the unit of the nodes number. The deployment area is the region where nodes can be distributed randomly. In the simulation experiments, the number of nodes is set from 100 to 140 with the step-size of 20 . The communication radius is the restricted condition for whether the two nodes are neighbor or not and it changes from $50 \mathrm{~m}$ to $80 \mathrm{~m}$ with the step-size of $5 \mathrm{~m}$.

Table 1 Several simulation parameters

\begin{tabular}{ccc}
\hline Simulation parameter & Indication & Value \\
\hline Deployment area $/ \mathrm{m}^{3}$ & $V$ & $200 \times 200 \times 200$ \\
Number of nodes $/ \mathrm{n}$ & $N$ & $100-140$ \\
Communication radius $/ \mathrm{m}$ & $R$ & $50-80$ \\
\hline
\end{tabular}

\subsection{Nodes deployment, nodes density, and varying of power levels}

When the simulation parameters are determined, nodes can be deployed. The nodes with different quantities are configured in the deployment area by using uniformly random distribution, respectively. Then in terms of different communication radii, the neighbor sets for all nodes are obtained, in other words, the rigid graph is acquired with different node quantities and communication radii by the approach of rigid block formation. An example with $N=100, V=200^{3} \mathrm{~m}^{3}, R=70 \mathrm{~m}$ is shown in Fig. 7 . It can be seen that there exist some nodes with the neighbor number being less than 4 , i.e., the graph is not global rigid, so it is necessary to adaptively increase $R$ until the graph becomes globally rigid. Node density is defined as the number of nodes per unit cube area. The relationship between node density and probability of the neighbor number $N_{i}\left(P_{N i}\right)$ equal to or greater than 4 is shown in Fig. 8, where the horizontal ordinate is node density while the vertical coordinate is the probability of the neighbor number equal to or greater than 4 .

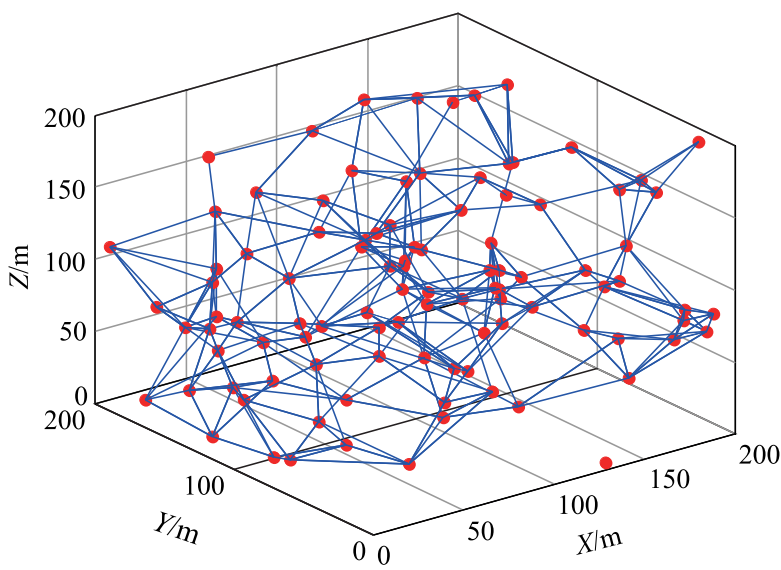

Fig. 7 An example with nodes of uniformly random distribution

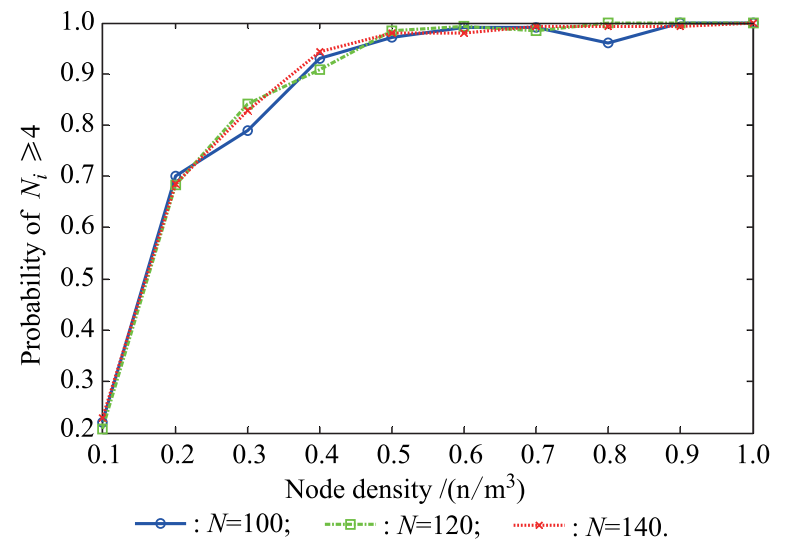

Fig. 8 Relationship between node density and probability of neighbor number $N_{i} \geqslant 4$

For the same node quantity, the probability is going up with the increasing of node density. Considering different node quantities, the tendency of the relation curve is consistent basically. The concrete data are shown in Table 2 . From Table 2, we can see that the probability is more than 0.9 when the node density is equal to $0.4 \mathrm{n} / \mathrm{m}^{3}$, while the probability reaches 1 when the node density is equal to or greater than $0.9 \mathrm{n} / \mathrm{m}^{3}$.

Table 2 Relationship between node density and probability of $N_{i} \geqslant 4$

\begin{tabular}{cccc}
\hline $\begin{array}{c}\text { Node density/ } \\
\left(\mathrm{n} / \mathrm{m}^{3}\right)\end{array}$ & $P_{N i}(100)$ & $P_{N i}(120)$ & $P_{N i}(140)$ \\
\hline 0.1 & 0.33 & 0.23 & 0.28 \\
0.2 & 0.56 & 0.65 & 0.79 \\
0.3 & 0.83 & 0.90 & 0.89 \\
0.4 & 0.92 & 0.94 & 0.91 \\
0.5 & 0.97 & 0.96 & 0.99 \\
0.6 & 0.98 & 0.98 & 0.99 \\
0.7 & 0.98 & 0.99 & 0.99 \\
0.8 & 0.97 & 1.00 & 1.00 \\
0.9 & 1.00 & 1.00 & 1.00 \\
1.0 & 1.00 & 1.00 & 1.00 \\
\hline
\end{tabular}

Therefore, the greater the node density is, the greater the probability of the neighbor number $N_{i} \geqslant 4$ is. What' $\mathrm{s}$ more, it is better to choose the value of node density equal to or greater than 0.4 .

\subsection{Node degree and average node degree}

The node degree is defined as the number of neighbors for each node with specific node quantity, distribution area and communication radius. From the previous subsection, the neighbor set for each node is obtained, so we can get the node degree for every node. The average node degree is defined as the ratio of the sum of node degree for each node to the number of WSN nodes $\left(N_{d}\right)$ deployed in the specific area. The simulation result is shown in Fig. 9 with $N=100$, the communication radius varies from $50 \mathrm{~m}$ to $75 \mathrm{~m}$ with the step-size of $5 \mathrm{~m}$, for each simulation set, 
there are ten instances. When $R$ is constant, the ten instances of average node degree are various, because ten instances have their own uniformly random distribution deployment. Although there exist differences, the values belong to an interval. Therefore, it is observed easily in the figure that the average node degree increases with the increasing of communication radius $R$. The reason is that as the increasing of communication radius, the average node degree augments at the same time, and the more rigid blocks can be acquired.

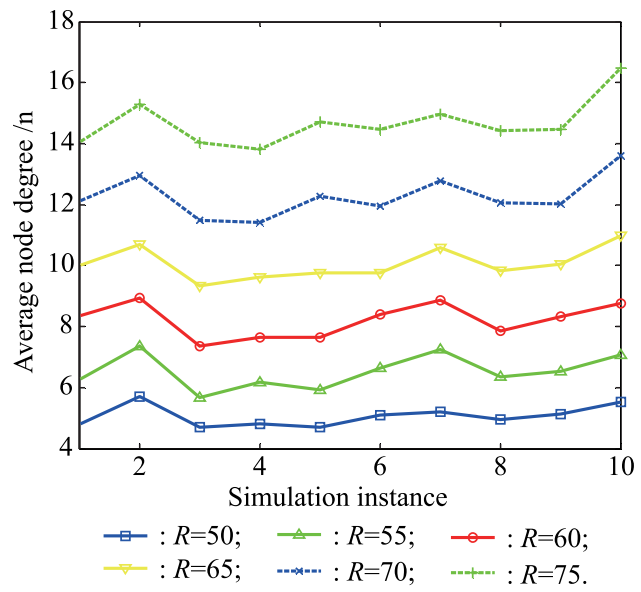

Fig. 9 Ten instances of $N=100$ with different $R$

When $N=100,120,140$, and communication radius increases from $50 \mathrm{~m}$ to $75 \mathrm{~m}$ with the step-size of 5 $\mathrm{m}$, there are 18 experiments. For each experiment, five average node degrees can be obtained, and the whole experimental results are shown in Table 3. It is clear that when $N=100,120,140$, the average node degree increases from 4.26 to $14.48,5.12$ to $18.22,6.09$ to 20.83 with different $R$, respectively. Therefore, we can obtain the same conclusion with Fig. 9 from the numerical data.

Table 3 Instances with different $N$ and $R$

\begin{tabular}{ccccccc}
\hline \multirow{2}{*}{$N / \mathrm{n}$} & \multicolumn{7}{c}{$R / \mathrm{m}$} \\
\cline { 2 - 7 } & 50 & 55 & 60 & 65 & 70 & 75 \\
\hline \multirow{6}{*}{100} & 4.26 & 5.82 & 7.12 & 9.1 & 10.94 & 12.84 \\
& 4.86 & 6.38 & 7.86 & 9.78 & 11.82 & 14.28 \\
& 4.96 & 6.08 & 7.46 & 9.36 & 11.06 & 13.54 \\
& 5.06 & 6.62 & 8.5 & 10.44 & 12.46 & 14.48 \\
& 5.12 & 6.48 & 8.18 & 10.1 & 11.68 & 14.1 \\
\hline \multirow{6}{*}{120} & 6.35 & 8.02 & 9.90 & 12.17 & 14.93 & 17.68 \\
& 6.27 & 7.88 & 9.68 & 12.40 & 15.08 & 18.22 \\
& 5.72 & 7.58 & 9.92 & 11.93 & 14.38 & 16.95 \\
& 6.47 & 8.02 & 10.13 & 12.33 & 15.25 & 17.88 \\
& 5.12 & 6.52 & 8.42 & 10.82 & 13.25 & 15.85 \\
\hline \multirow{6}{*}{140} & 6.56 & 8.46 & 10.84 & 12.96 & 15.83 & 18.77 \\
& 7.39 & 9.26 & 11.57 & 14.63 & 17.36 & 20.83 \\
& 6.66 & 8.60 & 10.70 & 13.23 & 15.94 & 18.77 \\
& 7.09 & 8.97 & 11.11 & 13.59 & 16.50 & 19.50 \\
& 6.09 & 7.94 & 10.23 & 12.70 & 15.06 & 17.77 \\
\hline
\end{tabular}

\subsection{Average node degree versus percentage of localizable nodes}

According to previous work in this paper, we know that when the neighbor number of a node is equal to or greater than 4 , we call this node localizable. The percentage of localizable nodes depends directly on the number of rigid blocks that can be generated for specific WSN deployment and a particular communication radius. The relationship between average node degree and percentage of localizable nodes is shown in Fig. 10. We can clearly see that when $N$ is fixed, the percentage of localizable nodes increases as the augment of average node degree. When average node degree is less than 5 , the localizable nodes are less than $60 \%$, while the average node degree is more than 10 , the localizable nodes are more than $90 \%$. The reason is that the greater average node degree is, the greater the number of rigid blocks can be obtained, and the easier localization does. Table 4 which is corresponding to Fig. 10 depicts the relationship between average node degree and percentage of localizable nodes. Some other conclusions can be obtained from Table 4. At the same $R$, as the number of nodes increases, the average node degree $\left(N_{\text {ave }}\right)$ and percentage of localizable nodes $\left(P_{L}\right)$ increase synchronously.

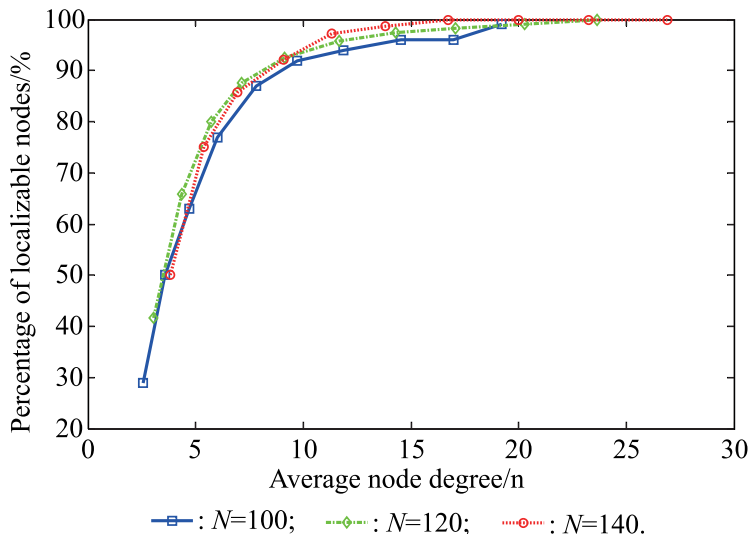

Fig. 10 Relationship between average node degree and percentage of localizable node

Table 4 Relationship between average node degree and percentage of localizable node

\begin{tabular}{ccccccccc}
\hline \multirow{2}{*}{$R / \mathrm{m}$} & \multicolumn{2}{c}{$N=100$} & & \multicolumn{2}{c}{$N=120$} & & \multicolumn{2}{c}{$N=140$} \\
\cline { 2 - 3 } \cline { 8 - 9 } \cline { 8 - 9 } & $P_{L} / \%$ & $N_{\text {ave }} / \mathrm{n}$ & & $P_{L} / \%$ & $N_{\text {ave }} / \mathrm{n}$ & & $P_{L} / \%$ & $N_{a v e} / \mathrm{n}$ \\
\hline 40 & 29.00 & 2.54 & & 41.67 & 3.03 & & 50.00 & 3.84 \\
45 & 50.00 & 3.56 & & 65.83 & 4.35 & & 75.00 & 5.39 \\
50 & 63.00 & 4.72 & & 80.00 & 5.73 & & 85.71 & 6.94 \\
55 & 77.00 & 6.00 & & 87.50 & 7.13 & & 92.14 & 9.09 \\
60 & 87.00 & 7.84 & & 92.50 & 9.12 & & 97.14 & 11.34 \\
65 & 92.00 & 9.74 & & 95.83 & 11.67 & & 98.57 & 13.79 \\
70 & 94.00 & 11.84 & & 97.50 & 14.30 & & 100.0 & 16.73 \\
75 & 96.00 & 14.56 & & 98.33 & 17.08 & & 100.0 & 20.01 \\
80 & 96.00 & 16.96 & & 99.17 & 20.28 & & 100.0 & 23.24 \\
85 & 99.00 & 19.20 & & 100.0 & 23.63 & & 100.0 & 26.90 \\
\hline
\end{tabular}


Combining Fig. 10 with Table 4, we can see that the curve corresponding to $N=140$ reaches $90 \%$ at first, and the curve corresponding to $N=100$ at last, i.e., the bigger $N$ is, the faster the curve of $P_{L}$ reaches. We also find that the three curves have different starting points.

Therefore, in order to get the greater percentage of localizable nodes, it is significant to magnify the average node degree to obtain more rigid quadrilateration blocks, and then to iterate localization. From the simulations, it is distinctively learned that the average node degree staying between 10 and 25 can acquire upper percentage of localizable nodes.

A comparison between our approach and the robust quads approach [17] which requires that the four referenced points are in a pentahedron and these points connect each other, is shown in Fig. 11. It is clear that no matter how much the average node degree is, the percentage of localization nodes by our approach is more than the robust quads approach. Therefore, our approach has greater performance than the robust quads approach.

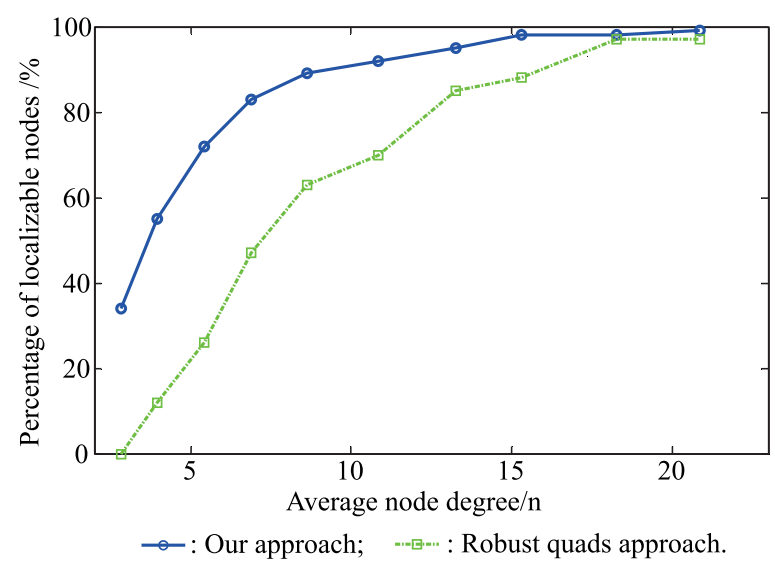

Fig. 11 A comparison between our approach and robust quads approach

\section{Conclusions}

This paper investigates the localization problem for WSNs in $3 \mathrm{D}$ space. We present a rigid graph-based algorithm that can divide the globally rigid graph into rigid blocks. The key point of this algorithm is to use the quadrilateration iteration technology to achieve the unique localizations of nodes in WSNs. Moreover, it has many advantages such as that it does not need fixed anchor nodes, the located node is unique, it is easy to realize and it does not depend on global coordinate. The simulations show some quantitative analysis and validate the effectiveness of the proposed algorithm. In fact, the localization problem of nodes for WSNs in 3D has not been well explored, such as the localizability, the 3D rigid graph generation and so on. Next, we will explore how to generate the 3D rigid graph in order to guarantee the localizability of WSNs.

\section{References}

[1] FADEL E, GUNGOR V C, NASSEF L. A survey on wireless sensor networks for smart grid. Computer Communications, 2015, 71(11): $22-33$.

[2] HERUTOMO A, ABDUROHMAN M, SUWASTIKA N A. Forest fire detection system reliability test using wireless sensor network and Open MTC communication platform. Proc. of the 3rd International Conference on Information and Communication Technology, 2015: 87-91.

[3] AHMED M, SALLEH M. Localization schemes in underwater sensor network (UWSN): a survey. Indonesian Journal of Electrical Engineering and Computer Science, 2016, 1(1): 119125.

[4] XU G, SHEN W, WANG X. Applications of wireless sensor networks in marine environment monitoring: a survey. Sensors, 2014, 14(9): 16932-16954.

[5] MALAVER A, MOTTA N, CORKE P. Development and integration of a solar powered unmanned aerial vehicle and a wireless sensor network to monitor greenhouse gases. Sensors, 2015, 15(2): $4072-4096$.

[6] XIAO F, LIU W, LI Z, et al. Noise-tolerant wireless sensor networks localization via multi-norms regularized matrix completion. IEEE Trans. on Vehicular Technology, 2017, 67(3): 2409-2419.

[7] HAN G, JIANG J, ZHANG C. A survey on mobile anchor node assisted localization in wireless sensor networks. IEEE Communications Surveys \& Tutorials, 2016, 18(3): 22202243.

[8] XIAO F, SHA C, CHEN L. Noise-tolerant localization from incomplete range measurements for wireless sensor networks. Proc. of the IEEE Conference on Computer Communications, 2015: 2794-2802.

[9] MAO G, FIDAN B, ANDERSON B D O. Wireless sensor network localization techniques. Computer Networks, 2007, 51(10): 2529-2553.

[10] SHRIVASTAVA A, BHARTI P. Localization techniques for wireless sensor networks. International Journal of Computer Applications, 2015, 116(12): 13-18.

[11] YIN J, WAN Q, YANG S. A simple and accurate TDOA-AOA localization method using two stations. IEEE Signal Processing Letters, 2016, 23(1): $144-148$.

[12] TOMIC S, BEKO M, RUI D. Distributed RSS-AoA based localization with unknown transmit powers. IEEE Wireless Communications Letters, 2016, 5(4): $392-395$.

[13] TOMIC S, BEKO M, RUI D. Distributed algorithm for target localization in wireless sensor networks using RSS and AoA measurements. Pervasive \& Mobile Computing, 2017, 37(6): $63-77$.

[14] HALDER S, GHOSAL A. A survey on mobile anchor assisted localization techniques in wireless sensor networks. Wireless Networks, 2015, 60(7): 1-20.

[15] HUANG B, XIE L, YANG Z. TDOA-based source localization with distance-dependent noises. IEEE Trans. on Wireless Communications, 2015, 14(1): $468-480$.

[16] JONDHALE S R, DESHPANDE R S, WALKE S M. Issues and challenges in RSSI based target localization and tracking in wireless sensor networks. Proc. of the International Conference on Automatic Control and Dynamic Optimization Techniques, 2016: $594-598$.

[17] MOORE D, LEONARD J, RUS D. Robust distributed network localization with noisy range measurements. Proc. of the In- 
ternational Conference on Embedded Networked Sensor Systems, 2004: 50-61.

[18] AHMADI R, EKBATANIFARD G, JAHANGIRY A. Improving localization in wireless sensor network using fixed and mobile guide nodes. Journal of Sensors, 2016: 6385380.

[19] XU H, SUN H, CHENG Y. Wireless sensor networks localization based on graph embedding with polynomial mapping. Computer Networks, 2016, 106: 151-160.

[20] KRISTALINA P, PRATIARSO A, BADRIYAH T. A wireless sensor networks localization using geometric triangulation scheme for object tracking in urban search and rescue application. Proc. of the 2nd International Conference on Science in Information Technology, 2016: 254-259.

[21] TOMIC S, MARIKJ M, BEKO M. Hybrid RSS-AoA technique for 3-D node localization in wireless sensor networks. Proc. of the International Wireless Communications and Mobile Computing Conference, 2015: 1277 - 1282.

[22] WEI N, GUO Q, SHU M L. Three-dimensional localization algorithm of wireless sensor networks base on particle swarm optimization. Journal of China Universities of Posts \& Telecommunications, 2012, 19(11): 7-12. (in Chinese)

[23] SHWE H Y, CHONG P H J. Cubic-based 3-D localization for wireless sensor networks. Proc. of the 9th International Conference on Information, Communications and Signal Processing, 2013: $169-179$.

[24] SECO F, JIMNEZ A R, ZHENG X. RFID-based centralized cooperative localization in indoor environments. Proc. of the International Conference on Indoor Positioning and Indoor Navigation, 2016: $1-7$.

[25] JI S. A time difference of arrival-based localization algorithm for wireless sensor networks. International Journal of Online Engineering, 2016, 12(11): 80-83.

[26] AHRIZ I, DZIRI A, RUYET D L. Compressed sensing-based centralized multiple targets localization. Proc. of the 11th International Symposium on Wireless Communications Systems, 2014: $563-567$.

[27] ZHU Y, GORTLER S J, THURSTON D. Sensor network localization using sensor perturbation. ACM Trans. on Sensor Networks, 2011, 7(4): 1-23.

[28] SHAMANTHA R B, VARMA S. An algorithmic approach to wireless sensor networks localization using rigid graphs. Journal of Sensors, 2016: 3986321.

[29] JACKSON B, JORDAN T. Connected rigidity matroids and unique realizations of graphs. Journal of Combinatorial Theory, 2003, 94(1): 1-29.

[30] TANIGAWA S I. Generic global rigidity of body-hinge frameworks. Journal of Combinatorial Theory, Series B, 2016, 117: $59-76$.

[31] MESBAHI M, EGERSTEDT M. Graph theoretic methods in multiagent networks. Princeton: Princeton University Press, 2010.
[32] LIU Y, YANG Z, WANG X, et al. Location, localization, and localizability. Journal of Computer Science and Technology, 2010, 25(2): 274-297.

[33] YANG Z, WU C, CHEN T. Detecting outlier measurements based on graph rigidity for wireless sensor network localization. IEEE Trans. on Vehicular Technology, 2013, 62(1): $374-$ 383.

\section{Biographies}

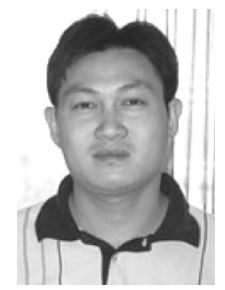

LUO Xiaoyuan was born in 1976 . He received his $\mathrm{Ph} . \mathrm{D}$. degree in control theory and control engineering from Yanshan University, China, Qinhuangdao, in 2005. He is currently a professor of the Institute of Electrical Engineering, Yanshan University. His current research interests include cooperative control of multi-agent systems and networked control systems.

E-mail: jiayao89@sina.cn

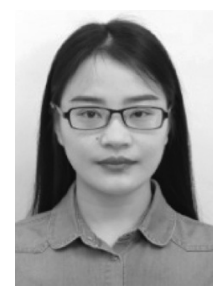

ZHONG Wenjing was born in 1994. She received her bachelor degree in automation from Shanxi University, China, in 2016. She is currently a master student in control theory and control engineering at Yanshan University. Her research interest mainly focuses on rigid graph-based localization for wireless sensor networks.

E-mail: 654110619@qq.com

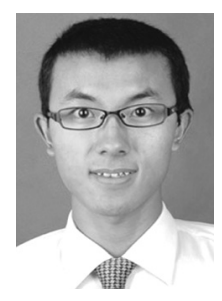

LI Xiaolei was born in 1989. He received his B.S. degree in automation from Yanshan University, Qinhuangdao, China, in 2014, and he is currently pursuing his Ph.D. degree in control science and engineering at Yanshan University. His current research interests include cooperative control of multi-agent systems and nonlinear control system.

E-mail: 505665918@163.com

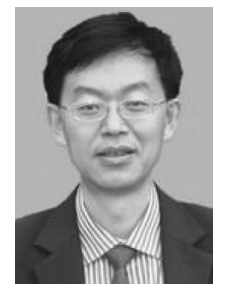

GUAN Xinping was born in 1963. He received his M.S. degree in applied mathematics and Ph.D. degree in electrical engineering from Harbin Institute of Technology, Harbin, China, in 1991 and 1999, respectively. He is currently a professor at Shanghai Jiao Tong University, Shanghai, China. His current research interests include networked control systems, wireless sensor networks and applications, and cooperative control of multi-agent systems.

E-mail: xpguan@sjtu.edu.cn 